\title{
Molecular pathogenesis of mantle cell lymphoma
}

\author{
Pedro Jares, Dolors Colomer, and Elias Campo \\ Hematopathology Section, Department of Pathology, Hospital Clinic, Institut d'Investigacions Biomèdiques August Pi i Sunyer, \\ University of Barcelona, Barcelona, Spain.
}

\begin{abstract}
Mantle cell lymphoma is a B cell malignancy in which constitutive dysregulation of cyclin D1 and the cell cycle, disruption of DNA damage response pathways, and activation of cell survival mechanisms contribute to oncogenesis. A small number of tumors lack cyclin D1 overexpression, suggesting that its dysregulation is always not required for tumor initiation. Some cases have hypermutated IGHV and stable karyotypes, a predominant nonnodal disease, and an indolent clinical evolution, which suggests that they may correspond to distinct subtypes of the disease. In this review, we discuss the molecular pathways that contribute to pathogenesis, and how improved understanding of these molecular mechanisms offers new perspectives for the treatment of patients.
\end{abstract}

\section{Introduction}

Mantle cell lymphoma (MCL) is a B cell malignancy with a broad spectrum of clinical, pathological, and biological features. The identification of the translocation event $\mathrm{t}(11 ; 14)(\mathrm{q} 13 ; \mathrm{q} 32)$ and the resulting cyclin D1 overexpression were of paramount importance in recognizing the clinical and biological diversity of this tumor. In addition to this constitutive dysregulation of the cell cycle, other mechanisms such as DNA damage response alterations and activation of cell survival pathways are integrated to drive MCL pathogenesis $(1,2)$. New observations are expanding our views on the ontogeny and pathogenesis of this lymphoma. Furthermore, these new insights into MCL oncogenesis are promoting the development of new therapeutic strategies, intended to target the molecular mechanism of the disease, and opening up new clinical perspectives for optimal diagnosis and management of the patients.

\section{Initial oncogenic steps}

The $t(11 ; 14)(\mathrm{q} 13 ; \mathrm{q} 32)$ translocation that juxtaposes the protooncogene CCND1 at 11q13 to the immunoglobulin heavy chain complex $(I G H)$ at chromosome $14 \mathrm{q} 32$ is considered the primary oncogenic mechanism in the development of MCL. This translocation forces the constitutive overexpression of cyclin D1, which is not detected in normal $\mathrm{B}$ lymphocytes, and deregulates the cell cycle at the $G_{1} / S$ phase transition (1). In addition to the primary translocation event, additional mechanisms that further increase cyclin D1 expression are frequently observed in MCL, underscoring its central importance in MCL lymphomagenesis. These mechanisms include secondary chromosomal rearrangement at $3^{\prime}$ of the CCND1 locus or mutations in the $3^{\prime}$ untranslated region ( $3^{\prime} \mathrm{UTR}$ ) that lead to the expression of truncated cyclin D1 transcripts missing part of the $3^{\prime}$ UTR $(3,4)$. These shorter transcripts, depleted of the destabilizing AU-rich elements and the binding sites for different microRNAs, have an extended half-life resulting in higher cyclin D1 protein levels and increased tumor aggressiveness $(4,5)$. Alternatively, increased overexpression of cyclin D1 can occur in MCL following the amplification of the translocated $\mathrm{t}(11 ; 14)$ allele $(6)$.

\section{Cell(s) of origin and ontogeny}

The initial translocation event $t(11 ; 14)(q 13 ; q 32)$ occurs at the pre-B stage of differentiation during the recombination of the

Conflict of interest: The authors have declared that no conflict of interest exists. Citation for this article: J Clin Invest. 2012;122(10):3416-3423. doi:10.1172/JCI61272.
$\mathrm{V}(\mathrm{D}) \mathrm{J}$ segments of the IGH variable region (IGHV) in the bone marrow. However, the tumor is composed of a specific population of mature B lymphocytes, indicating that the full neoplastic phenotype is acquired at later stages of the $\mathrm{B}$ cell differentiation process (7). Historically, naive B cells have been considered the normal counterpart to MCL tumor cells based on $\operatorname{IgM} / \operatorname{IgD}$ and CD5 expression by the tumor cells, their topographic distribution in the mantle zones, and early descriptions of the predominant use of unmutated IGHV. More recently, comprehensive analysis of B cell receptor (BCR) diversity in MCL has shifted this view to a more complex ontogenetic model, in which antigen selection plays an important role in pathogenesis, at least for a subset of tumors. Recent studies have shown that $15 \%-40 \%$ of MCLs carry $I G H V$ hypermutations, with a strong bias in the IGHV gene repertoire (8-10). As is the case for chronic lymphocytic leukemia (CLL), stereotyped heavy complementarity-determining region 3 (VH CDR3) sequences have been recognized in 10\% of MCL. Although the stereotyped subsets are clearly distinct from those described in CLL, their existence suggests a strong role of antigendriven selection in the clonogenic expansion of MCL tumor cells. These findings open a complex scenario with more than one possible cell subtype dominating in different subtypes of MCL (Figure 1). In the absence of $I G H V$ mutations, MCL may still derive from naive B cells, but cases with stereotyped BCR are likely antigen selected. Furthermore, MCL carrying a high mutational load may originate from cells strongly influenced by the germinal center microenvironment. Finally, the progenitor cells of cases with a low number of somatic mutations may derive from cells of the marginal zone, intermediate cells between naive and germinal center cells already expressing AID or transitional B cells resembling murine B-1 B cells $(11,12)$.

\section{Genetically deregulated oncogenic pathways}

The role of cyclin D1 in promoting MCL lymphomagenesis is related to its function in the cell cycle regulating the cyclin-dependent kinases CDK4 and CDK6. Cyclin D1 binding to CDK4/6 activates the transcription factor E2F by phosphorylating its inhibitor, retinoblastoma 1 (RB1), and further promotes cyclin E/CDK2 activation to trigger entry into the $\mathrm{S}$ phase of the cell cycle (1).

Cyclin D1 may have additional oncogenic effects beyond its role in the cell cycle. Studies in solid tumor models have shown cyclin D1 in transcription regulation as interacting with transcription factors, chromatin-remodeling, and histone-modifying enzymes (13-15). Cyclin D1 may also promote chromosome instability 


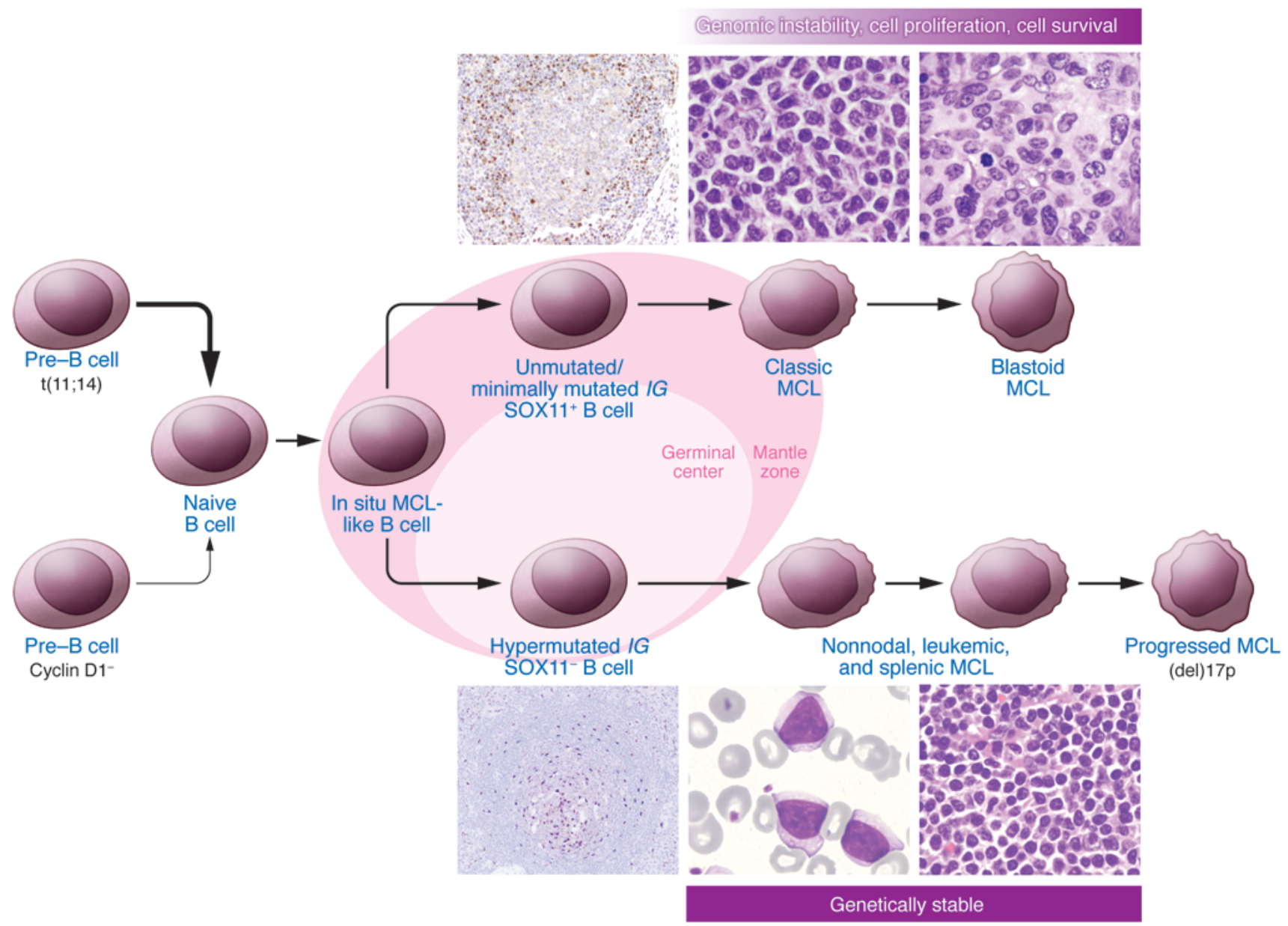

Figure 1

Hypothetical models of two different molecular subtypes of MCL. The naive B cell carrying the $t(11 ; 14)$ colonizes the mantle zone of the lymphoid follicle and generates an in situ MCL lesion. Most MCLs evolve from these cells or cells in the marginal zone with no or limited IGHV somatic mutations. These tumors express SOX11, are genetically unstable, and tend to accumulate alterations in genes dysregulating cell cycle, DNA damage response pathways, and cell survival mechanisms. Alternatively, some cells with the $t(11 ; 14)$ may enter the germinal center and undergo IGHV somatic hypermutations. These cells are genetically stable and do not express SOX11. The tumors derived from these cells tend to spread to the peripheral blood and spleen more than to lymph nodes. The disease seems to be stable for long periods of time, but some of these tumors may acquire mutations in genes such as TP53 that lead to disease progression. Intriguingly, some tumors with the pathological and genetic features of MCL do not carry translocations of the CCND1, CCND2, and CCND3.

by binding to genes that regulate chromosome segregation and chromatin reorganization (16). Intriguingly, cyclin D1 has been implicated in promoting DNA repair by binding to RAG1 and homologous DNA recombination (17). How this DNA-repairing function reconciles with the cyclin D1 oncogenic role is not clear, but it may help the cells to survive under DNA-damaging oncogenic stress or the action of genotoxic drugs (18). These non-cell cycle-related functions of cyclin D1 have not been properly investigated in MCL. However, the complete inactivation of the RB1 by mutations and deletions in some MCLs (19), making cyclin D1 dispensable for cell cycle functions, would support the idea that cyclin D1 may play additional oncogenic roles in these tumors. Interestingly, the recent description of cyclin D1 as promoting cell survival in MCL by sequestering the proapoptotic BAX protein reinforces this concept (20).

Several observations suggest that cyclin D1 dysregulation is not sufficient for cell transformation, nor does it explain the aggres- sive behavior of MCL. Secondary chromosome alterations that target genes involved in molecular pathways, such as cell cycle control, DNA damage response, and cell survival pathways, are frequently found in aggressive MCL. The INK4a/CDK4/RB1 and ARF/MDM2/p53 cell cycle pathways are very frequently targeted by secondary genetic alterations in MCL. Both pathways are connected through the CDKN2A locus $(9 \mathrm{p} 21)$, which encodes for both the CDK inhibitor INK4a and the positive p53 regulator ARF, and this locus is frequently deleted in MCL. Other key elements of these pathways, such as TP53 and RB1, are also frequently inactivated by point mutations or gene deletions (19, 21). In addition, gene amplification events have been found to deregulate additional genes, including $C D K 4$, polycomb ring finger gene BMI1, and MDM2 $(19,22)$. Accordingly, the proliferation gene expression signature is the best predictor of patient survival, underscoring the importance of cell cycle dysregulation in dictating the behavior of MCL (23). 


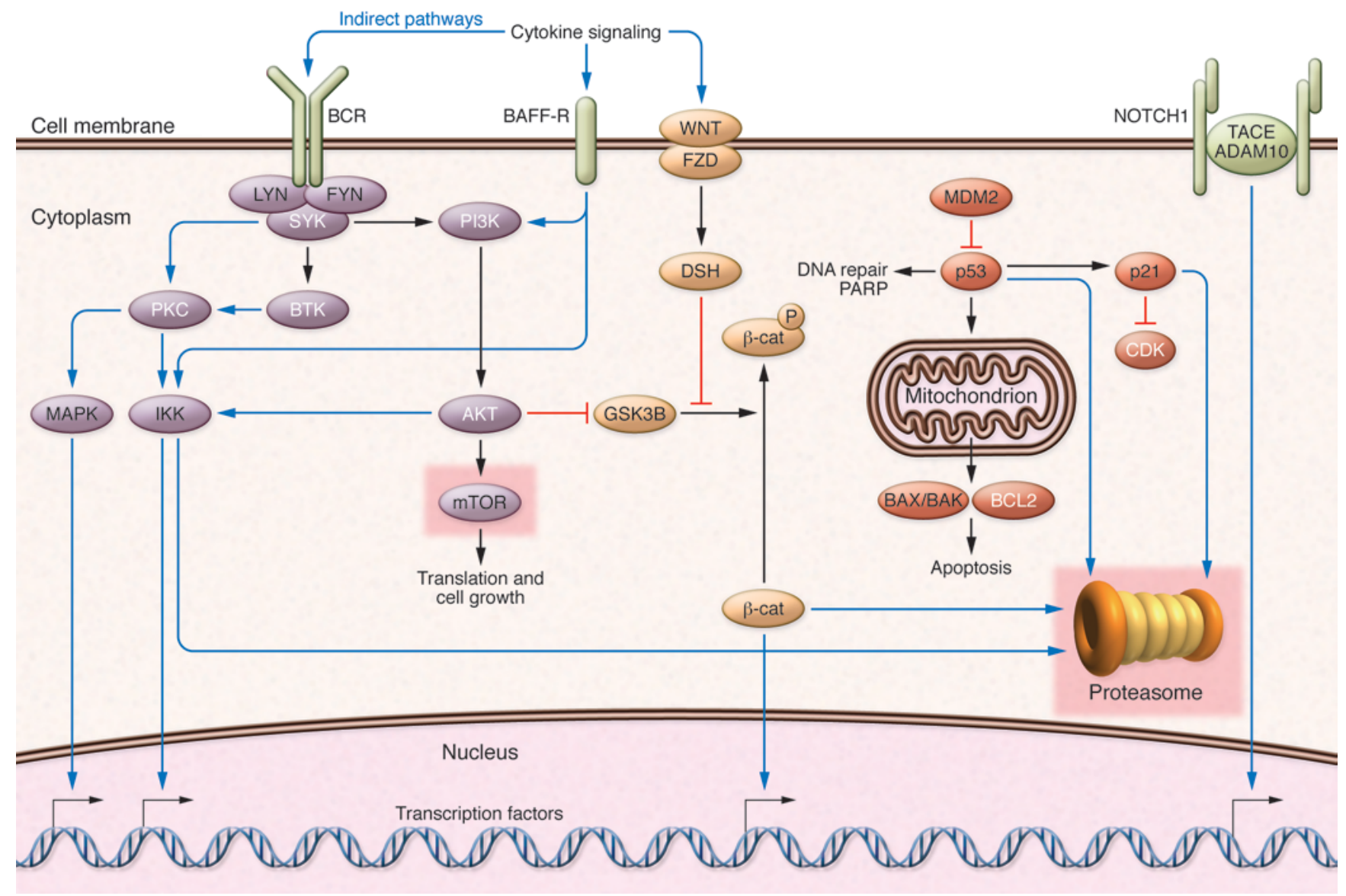

Figure 2

Major aberrant pathways in MCL susceptible to targeted therapies. Numerous signaling pathways are constitutively activated and/or deregulated in MCL, including BCR, BAFF-R, mTOR, WNT, and NOTCH1 signaling as well as pathways that promote the cell cycle and inhibit apoptosis. mTOR and proteasome inhibitors are the only two pathways for which specific drugs have been approved. Several small molecules targeting the $\mathrm{BCR}$ and the PI3K/AKT/mTOR pathway at different levels (indicated by white text) are currently being studied in clinical trials. Other clinical trials are using small molecules to target the BCL2 family proteins directly involved in apoptosis and the cyclin-dependent kinases (CDKs) directly involved in the progression through $\mathrm{G}_{1} / \mathrm{S}$ and $\mathrm{G}_{2} / \mathrm{M}$ phases of the cell cycle. The WNT and NOTCH1 pathways are potential targets for $\beta$-catenin ( $\beta$-cat) inhibitors and NOTCH1 inhibitors, respectively. Pharmacologic inhibition of PARP activity has become an interesting therapeutic strategy in tumors with dysfunctional DNA repair mechanisms, such as MCL.

Chromosome aberrations observed in MCL are consistent with an important role for dysregulation of the DNA damage response in this lymphoma (24). The ataxia telangiectasia mutated (ATM) gene, located at 11q22-23, is frequently deleted and mutated in MCL cases with increased genomic instability (25). Additional downstream elements of this pathway, including CHK1 and CHK2, are occasionally deregulated in MCL, suggesting that mutation of DNA damage response contributes to oncogenesis (1). Recent studies have also shown that genes involved in cell survival are targets of recurrent genetic alterations in MCL. Amplifications and overexpression of antiapoptotic genes such as BCL2 (18q21) (6) and homozygous deletions of proapoptotic genes such as BCL2L11 (2q13) have been described in primary tumors (26).

A recent study found NOTCH1 mutations in $12 \%$ of MCL cases to be associated with poor survival (27). This gene encodes for a transmembrane protein that functions as a ligand-activated transcription factor. Similarly to NOTCH1 mutations described recently in CLL $(28,29)$, mutations in MCL occur in the PEST domain and generate a truncated, more stable and transcriptionally active protein. In the study mentioned above, inhibition of this pathway reduced proliferation and induced apoptosis of MCL cells (27).

\section{Cyclin D1 may be dispensable for MCL pathogenesis}

The existence of uncommon lymphomas with pathological features of MCL in the absence of cyclin D1 dysregulation suggests that cyclin D1 overexpression is not the only mutation that can initiate MCL development (Figure 1). These tumors have a similar gene expression profile and share the same type of secondary chromosomal alterations, supporting the idea that they correspond to the same molecular disease. Chromosomal translocations fusing the CCND2 gene, but not the CCND3 gene, to IG loci are found in $50 \%$ of the cases, suggesting that this may be an alternative mechanism to CCND1 translocation $(30,31)$. However, the absence of any cyclin $\mathrm{D}$ gene alterations in a subset of otherwise conventional MCL raises the intriguing question about the initial driver for pathogenesis in these cases. 


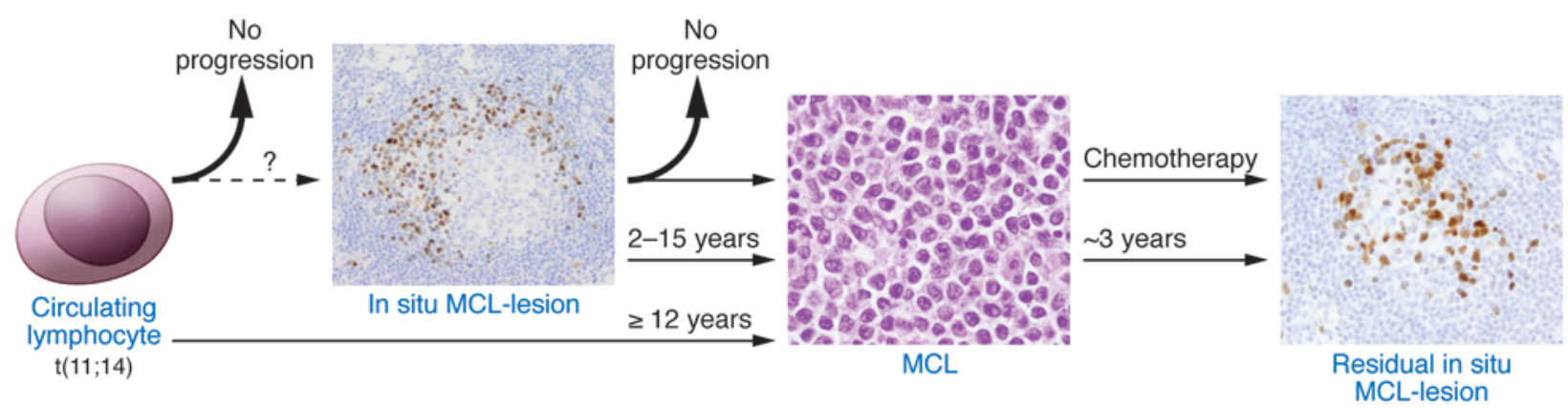

Figure 3

Multistep model in the progression of MCL. Clonal cells carrying the $t(11 ; 14)$ translocation may be detected in the peripheral blood of healthy individuals at very low levels. The risk of progression of these clones must be extremely low, if any. Cells expressing cyclin D1 and carrying the $t(11 ; 14)$ may be found in the mantle zone of lymphoid follicles in otherwise reactive tissues. Most of these lesions will not evolve into an overt lymphoma. The incidental detection of tumor cells in the mantle zone of reactive tissues in patients with MCL who appear to be in complete remission suggests that this microenvironment may sustain chemoresistant cells. Anecdotal clinical observations provide a timeframe for the potential evolution of these lesions. Original magnification, $\times 100$ (right and left), $\times 400$ (center).

In a search for new genes relevant in MCL pathogenesis, SOX11, a neuronal transcription factor, was identified as a very specific marker of MCL (32). SOX11 is highly expressed in virtually all MCL tumors but is not expressed in other mature lymphoid neoplasms and normal lymphocytes at any stage of differentiation. Interestingly, SOX11 is highly expressed in both cyclin D1-negative and-positive MCL, suggesting that in addition to its value as a diagnostic biomarker, it may be an important factor in the pathogenesis of MCL (33). SOX11 expression in normal and neoplastic lymphoid cells is regulated by epigenetic changes, but its function in tumor development is not well understood yet (34). Further studies of potential oncogenic mechanisms common to cyclin D1-positive and -negative MCL may provide new insights into the primary genetic events that drive the development of one of the most aggressive lymphomas.

\section{Signaling pathways deregulated in MCL}

In addition to the multiple genetic alterations observed in MCL, these tumors carry dysregulation of different signaling pathways involved in cell proliferation, survival, and interactions with the microenvironment. Some of them are beginning to be targeted with new therapies and open a new perspective for the treatment of patients (Figure 2).

The PI3K/AKT/mammalian target of rapamycin (PI3K/AKT/ $\mathrm{mTOR}$ ) pathway plays a critical regulatory role in cell growth and death processes. The causes for the activation of this pathway are not well understood but may include BCR, tumor necrosis factor (ligand) superfamily, member 13b (BAFF), and CD40 signaling. High levels of activated AKT, mTOR, and their respective downstream targets have been identified in MCL (35-37). Loss of expression or inactivating phosphorylation of PTEN, a negative PI3K regulator, have been found in some MCLs associated with the constitutive activation of AKT (37). No genetic or epigenetic alterations of PTEN have been identified in MCL, but the microRNA (miRNA) miR17-92 cluster, which is frequently amplified and overexpressed in MCL (38), was recently shown to target the PTEN transcript (39). Overexpression of these miRNAs causes downregulation of PTEN and activation of the AKT pathway and promotes resistance to chemotherapy-induced apoptosis in these tumors (39).
The influence of the microenvironment in the pathogenesis of different lymphoid neoplasms is receiving increased interest, but its potential role in MCL has been less investigated. The recent studies of the BCR supporting an antigen selection in the pathogenesis of at least a subset of MCL suggest a role for the stimulation of this pathway in the clonal expansion of tumor cells. However, the specific mechanisms and downstream effectors potentially involved are not well known. Amplification and overexpression of the spleen tyrosine kinase (SYK) and the PI3K catalytic $\alpha$ polypeptide have been found in a subset of MCL $(40,41)$. Furthermore, different kinases of the BCR signaling, including SYK, LYN, and $\mathrm{BTK}$, have been found to be phosphorylated in primary MCL, suggesting activation of the pathway (42).

Activation of the JAK/STAT pathway may be a relevant mechanism connecting external stimuli with cell survival and proliferation. Phosphorylated STAT3 has been observed in primary MCL $(43,44)$. This activation may occur through a cytokine-dependent autocrine IL- 6 and/or IL-10 secretion loop and/or induced upon BCR engagement (43). The IL-10 receptor is overexpressed in some MCLs (45), and IL-10 has been shown to sustain cell proliferation in MCL primary cells (46). Inactivating mutations of SOCS1, an inhibitor of the JAK/STAT pathway, have been described in some MCLs (47). Interestingly, STAT3 activation seems to be more common in MCLs with hypermutated (compared with unmutated) $I G H V$, suggesting that these two subtypes of MCL may use different cell survival mechanisms (43).

Expression of CD40 and responsiveness to the CD40 ligand (CD154) (48) suggest that MCL cells can interact with CD154positive $\mathrm{T}$ cells in tissues, where such cellular interactions could promote MCL cell survival and expansion (49). High levels of functional CXCR4, CXCR5, and VLA-4 (CD49d) have been reported, suggesting a role in the migratory properties of the tumor cells (50). Further, the IL-22 receptor is aberrantly expressed in MCL, and stimulation by IL-22 promotes tumor cell growth and survival (51).

Constitutive activation of NF- $\mathrm{KB}$ has been detected in MCL cell lines and primary tumors with overexpression of several target genes, including the antiapoptotic proteins CFLIP, BCL2, BCLXL, and XIAP $(52,53)$. BCR signaling, microenvironmental stimuli, and TNF signaling may mediate NF- $\mathrm{KB}$ activation. Inactivation of the NF-KB pathway inhibitors TNFAIP3/A20 (6p23) and FAF1 
(1p32.3) by mutations and homozygous deletions, respectively, have been reported in primary $\operatorname{MCL}(26,54)$.

The WNT canonical pathway has been found to be deregulated in MCL, with nuclear localization of $\beta$-catenin in primary tumors $(36,55)$. Concordantly, inactive phospho-GSK3B, a key component of the destruction complex responsible for $\beta$-catenin inactivation, was also found in MCL, suggesting that the WNT pathway may play a role in MCL pathogenesis. However, further studies are needed to confirm and expand these observations.

\section{Are all MCLs created equal? An alternative pathogenetic pathway with clinical implications}

MCL is classically considered an aggressive lymphoma. However, some studies have described a subset of patients with an indolent evolution of MCL even in the long-term absence of chemotherapy. The identification of these patients is puzzling given the current understanding of MCL as an aggressive tumor, which leads to the frequent recommendation of immediate treatment in almost all patients. These patients may benefit from a "watch and wait" management approach, at least for some period of time, but the challenge is in distinguishing them from patients who need aggressive therapy as soon as the disease is identified $(56,57)$.

MCLs with a very low proliferation index, a histological growth pattern restricted to the mantle zone of the lymphoid follicles (mantle zone pattern), and a limited clinical stage may have a more indolent behavior than MCLs without these features (58-61). These cases may correspond to early stages in the development of conventional MCL.

In addition, clinical studies have recognized a subgroup of MCL patients with an indolent behavior who, contrary to most cases of conventional MCL, present with a predominantly leukemic nonnodal disease. The genetic and molecular mechanisms underlying this distinct biological behavior are not fully understood, but recent studies comparing the groups of indolent nonnodal and conventional MCLs have revealed marked differences between the two (Figure 1). Most MCLs have complex karyotypes, but the nonnodal tumors have very few, if any, chromosomal alterations in addition to the $\mathrm{t}(11,14)$ translocation. The nonnodal MCLs frequently have hypermutated $I G H V$, suggesting an origin in cells with a strong influence of the germinal center microenvironment, whereas conventional MCLs typically have few or no mutations in IGHV (refs. 62-64 and Figure 1). The gene expression profiles of these two subtypes of MCL show differences in certain gene signatures. The indolent tumors have very low or no expression of a subset of genes of the high-mobility group of transcription factors, including SOX11, that are highly expressed in conventional tumors (65). Nonnodal tumors also have downregulation of genes related to the DNA damage pathway and genes associated with cell adhesion (63).

The detection of SOX11 by immunohistochemistry or quantitative PCR in larger series of patients has confirmed the relationships among its lack of expression, hypermutated $I G H V$, low karyotype complexity, nonnodal leukemic disease, and longer survival with stable disease in independent cohorts of patients, suggesting that these biological and clinical features may identify a different subtype of MCL (63-67). Some patients with nonnodal disease may progress to an aggressive form of MCL after several years of a stable clinical course. In addition, some SOX11-negative tumors may progress rapidly after diagnosis and have a poor prognosis $(61,64)$. Interestingly recent stud- ies have shown that the aggressive behavior of SOX11-negative MCL seems related to an extensive nodal disease, blastoid morphology, complex karyotypes, and 17p/TP53 alterations $(61,64$, 68). These observations suggest that these aggressive tumors may correspond to a transformed phase of the SOX11-negative MCL (Figure 1). Therefore, SOX11 expression by itself should not be considered a prognostic parameter but a biomarker that may help to recognize a particular subtype of MCL with different clinical and biological features than conventional MCL. The SOX11-negative tumors may have a long leukemic, nonnodal phase followed in some cases by the progression to an aggressive lymphoma associated with the acquisition of $17 \mathrm{p} / \mathrm{TP} 53$ alterations and complex karyotypes. Given the asymptomatic nature of the leukemic phase, some patients may only be diagnosed at the end of this process, when the extensive nodal disease develops. We recently proposed the term "nonnodal type of MCL" for this subtype of MCL (64) because it reflects the main, although not entirely specific clinical feature and because it gives credit to the initial clinical observations of this subgroup of tumors $(62,69)$.

\section{Multistep development and progression in MCL}

The contribution of multiple mutations to tumor development is not as well characterized in lymphoid neoplasms as in solid tumors. Recent observations have identified what may correspond to early molecular and pathological phases of MCL oncogenesis (Figure 3). Cells carrying the $\mathrm{t}(11 ; 14)$ translocation have been detected at very low levels in the peripheral blood of a number of healthy individuals (8\%). These clones can persist for long periods, but their potential to evolve into an overt lymphoma is not clear (70). Their high frequency in healthy individuals and low prevalence of MCL suggests that most clones bearing only the $t(11 ; 14)$ translocation will never transform into a malignant tumor. On the other hand, the observation of a simultaneous MCL with the same clonal origin in a recipient and donor 12 years after an allogenic bone marrow transplant underscores the long latency required by initial clones to develop an overt lymphoma (71).

Cells carrying the $t(11 ; 14)$ translocation and cyclin D1 overexpression have been occasionally found in the mantle zones of otherwise reactive lymphoid tissues in healthy individuals. These lesions have been referred to as "in situ MCLs," but their malignant potential seems very limited (Figures 1 and 3). In a recent study, only 1 of 12 of these lesions developed an overt MCL four years after its detection. To avoid overtreatment, the alternative term "in situ MCL-like B cells" has been proposed (72). Retrospective analysis in seven patients identified in situ lesions in tissues obtained 2 to 15 years prior to diagnosis, suggesting that all MCLs proceeded through a stage of in situ lesions (73). Some in situ lesions express SOX11, whereas others are SOX11 negative, suggesting that the in situ lesion stage may be a common step in both SOX11negative and -positive subtypes of MCL (ref. 71 and Figure 1). Incidents of in situ lesions have also been found in patients who are apparently in complete remission following treatment, suggesting that this microenvironment may sustain residual tumor cells resistant to chemotherapy (ref. 71 and Figure 3).

\section{Target therapy in MCL: a promise of more successful treatments}

The heterogeneous biology and clinical presentation of MCLs are major challenges to define standard therapies (74). Recent protocols adjusted for the patients' conditions are improving the 
clinical outcome, but frequent relapses and progressive resistance to treatment emphasize the need for new approaches (75). The increasing understanding of the pathogenetic mechanisms provides attractive and promising targets. In the last six years, two new drugs for relapsed or refractory MCL have been approved: the proteasome inhibitor bortezomib (approved by the US FDA; ref. 76) and the mTOR inhibitor temsirolimus (approved by the European Medicines Agency; ref. 77.

Proteasome inhibitors disrupt the ubiquitin-proteasome system (78). Bortezomib induces apoptosis in MCL through upregulation of the BH3-only protein NOXA (79). NOXA acts as a downstream effector of an integrated cellular stress response triggered by the accumulation of undegraded, polyubiquitinated proteins that induce endoplasmic reticulum stress and the generation of reactive oxygen species (80). Single-agent bortezomib therapy was shown to induce responses in $30 \%-50 \%$ of cases, even in patients who failed to respond to prior dose-intensive therapy or who had bulky disease (81). A second generation of proteasome inhibitors, including NPI-0052 (salinosporamide A), PR-171 (carfilzomib), and MLN9708, are now entering the clinic. Efficacy has also been reported for proteasome inhibitors in combination with other cytotoxic agents and targeted therapies such as histone deacetylase inhibitors and HSP90 inhibitors $(78,82)$. Several proteins central to MCL, including cyclin D1, CDK4, AKT, and p53, bind to the molecular chaperone HSP90, suggesting that HSP90 inhibitors may be a suitable therapeutic target (83). However, HSP90 inhibitors as well as histone deacetylase inhibitors have demonstrated only moderate clinical antitumor activity when used alone $(78,82,84)$.

$\mathrm{mTOR}$ is another attractive target for new therapies (85). Temsirolimus is a rapamycin analog that allosterically inhibits mTORC1. As a single agent, temsirolimus was shown to induce responses in up to $40 \%$ of relapsed or refractory patients, and its efficacy was superior to that of traditional monotherapies (86). Phase I/II studies are currently being performed to explore the best chemotherapy that may be added to temsirolimus and its potential role as maintenance therapy (87-92). Other rapalogs, such as everolimus and deferolimus, and several PI3K inhibitors offer additional means to target the PI3K/AKT/mTOR pathway, although deferolimus has not showed clinical activity in relapsed $\operatorname{MCL}(93,94)$. The PI3K- $\delta$ inhibitor CAL101 has shown an overall response rate of $62 \%$ in relapsed and refractory MCL cases (95). In addition, several ongoing clinical trials are using MK2206, an allosteric inhibitor of AKT, and OSI-027, a dual mTORC1 and mTORC2 inhibitor $(82,96)$.

Some therapies target cell cycle regulators and other key pathogenetic pathways in MCL $(22,97,98)$. The pan-CDK inhibitor flavopiridol has shown minimal antitumoral activity (99), but in contrast PD-0332991, an inhibitor of CDK4 and CDK6, has shown biological effects (reduced retinoblastoma phosphorylation and $\mathrm{Ki} 67$ index) and some clinical benefit in a subset of patients (100). Promising results are being obtained with drugs targeting the DNA repair pathway $(25,101)$, such as the poly(ADPribose) polymerase 1 (PARP) inhibitor AG014699 (102-104). The use of proapoptotic molecules that mimic the activity of BH3-only proteins (105) has shown promising Results in preclinical models (106-108) but no responses or only modest activity in clinical studies $(82,109)$. However, these compounds may sensitize cells to the effect of other therapeutic agents.

Novel approaches include targeting the BCR pathway. Unfortunately, PKCB inhibitors showed no objective responses in
MCL patients (110), and an objective response to SYK inhibitors was reported in only $11 \%$ of patients (111). In contrast, the BTK inhibitor PCI-32765 (ibrutinib) induced a high rate of objective responses in patients with relapsed or refractory MCL $(111,112)$. In addition, a Phase I clinical trial using the oral JAK-2 inhibitor SB1518 showed activity in two of three MCL cases studied (113). The identification of recurrent NOTCH1 mutations provided a preclinical rationale for therapeutic inhibition of the NOTCH pathway using $\gamma$-secretase inhibitors or for immunotherapy using antibodies against NOTCH1 $(114,115)$.

\section{Conclusions and future directions}

The study of MCL, integrating multidisciplinary research from molecular biology and pathology to the clinic, has improved the understanding of its pathogenesis and guides the search to design new therapeutic strategies. Although advances in the last decade have been remarkable, recent clinical and biological observations have shown that our understanding of this tumor is far from complete. The increasing evidence for a role for cyclin D1 beyond cell cycle in some tumor models raises the question of similar functions in MCL. On the other hand, the absence of cyclin D1/D2 dysregulation in some cases argues for additional mechanisms in the initiation of the tumor. The intricate networks of genetic alterations, deregulated signaling pathways, and their triggering elements are only starting to be elucidated. The identification of two subtypes of the disease sharing the cyclin D1 dysregulation but with different molecular, genetic, and clinical characteristics and evolution is intriguing and requires further study. The role of antigen selection and different cells of origin for these subtypes of MCL may highlight the influence of the microenvironment in the development of the disease. The investigation of these new observations should render a better understanding of the tumor and facilitate a more precise diagnosis and tailored management of patients, particularly in light of an increasing number of new potential therapeutic drugs.

\section{Acknowledgments}

We gratefully acknowledge M. Pinyol, L. Hernandez, S. Bea, P. Perez-Galan, G. Roue, V. Amador, J.I. Martin-Subero, and the rest of the members of our group for the critical feedback and thoughtful discussions. We acknowledge A. Rosenwald and G. Ott from the University of Würzburg and our colleagues from the European Mantle Cell Lymphoma Network and the Mantle Cell Lymphoma Consortium of the Lymphoma Research Foundation for their continuous collaboration on these projects. The authors are supported by the Spanish Ministry of Economy and Competitivity (grants SAF 2008-03630 to E. Campo and SAF 2009-09503 to D. Colomer), the Instituto de Salud Carlos III, the Red Temática de Investigación Cooperativa de Cáncer (RTICC; grants RD06/0020/0039 to E. Campo and RD06/0020/0014 to D. Colomer), the Generalitat de Catalunya (grants 2009-SGR-992 to E. Campo and 2009-SGR-967 to D. Colomer); and the Mantle Cell Lymphoma Consortium of the Lymphoma Research Foundation.

Address correspondence to: Elias Campo, Hematopathology Section, Department of Anatomic Pathology, Hospital Clinic, Institut d'Investigacions Biomediques August Pi i Sunyer (IDIBAPS), Universidad de Barcelona, Villarroel 170, 08036-Barcelona, Spain. Phone: 34932275450; Fax: 34932275572; E-mail: ecampo@clinic.ub.es. 
1. Jares P, Colomer D, Campo E. Genetic and molecular pathogenesis of mantle cell lymphoma: perspectives for new targeted therapeutics. Nat Rev Cancer. 2007;7(10):750-762.

2. Jares P, Campo E. Advances in the understanding of mantle cell lymphoma. Br J Haematol. 2008; 142(2):149-165.

3. Chen RW, et al. Truncation in CCND1 mRNA alters miR-16-1 regulation in mantle cell lymphoma. Blood. 2008;112(3):822-829.

4. Wiestner A, et al. Point mutations and genomic deletions in CCND1 create stable truncated cyclin D1 mRNAs that are associated with increased proliferation rate and shorter survival. Blood. 2007 109(11):4599-4606.

5. Slotta-Huspenina J, et al. The impact of cyclin D1 mRNA isoforms, morphology and p53 in mantle cell lymphoma: p53 alterations and blastoid morphology are strong predictors of a high proliferation index. Haematologica. 2012;97(9):1422-1430.

6 . Bea S, et al. Uniparental disomies, homozygous deletions, amplifications, and target genes in mantle cell lymphoma revealed by integrative highresolution whole-genome profiling. Blood. 2009; 113(13):3059-3069.

7. Welzel N, et al. Templated nucleotide addition and immunoglobulin JH-gene utilization in $\mathrm{t}(11 ; 14)$ junctions: implications for the mechanism of translocation and the origin of mantle cell lymphoma. Cancer Res. 2001;61(4):1629-1636.

8. Walsh SH, et al. Mutated VH genes and preferential VH3-21 use define new subsets of mantle cell lymphoma. Blood. 2003;101(10):4047-4054

9. Kienle D, et al. VH mutation status and VDJ rearrangement structure in mantle cell lymphoma: correlation with genomic aberrations, clinical characteristics, and outcome. Blood. 2003;102(8):3003-3009.

10. Hadzidimitriou A, et al. Is there a role for antigen selection in mantle cell lymphoma? Immunogenetic support from a series of 807 cases. Blood. 2011; 118(11):3088-3095.

11. Kolar GR, Mehta D, Pelayo R, Capra JD. A novel human B cell subpopulation representing the initial germinal center population to express AID. Blood. 2007;109(6):2545-2552.

12. Sims GP, Ettinger R, Shirota Y, Yarboro CH, Illei GG, Lipsky PE. Identification and characterization of circulating human transitional B cells. Blood. 2005;105(11):4390-4398.

13. Fu M, Wang C, Li Z, Sakamaki T, Pestell RG. Minireview: Cyclin D1: normal and abnormal functions. Endocrinology. 2004;145(12):5439-5447.

14. Aggarwal P, et al. Nuclear cyclin D1/CDK4 kinase regulates CUL4 expression and triggers neoplastic growth via activation of the PRMT5 methyltransferase. Cancer Cell. 2010;18(4):329-340.

15. Bienvenu F, et al. Transcriptional role of cyclin D1 in development revealed by a genetic-proteomic screen. Nature. 2010;463(7279):374-378.

16. Casimiro MC, et al. ChIP sequencing of cyclin D1 reveals a transcriptional role in chromosomal instability in mice. J Clin Invest. 2012;122(3):833-843

17. Jirawatnotai $S$, et al. A function for cyclin D1 in DNA repair uncovered by protein interactome analyses in human cancers. Nature. 2011;474(7350):230-234.

18. Bartek J, Lukas J. DNA repair: Cyclin D1 multitasks. Nature. 2011;474(7350):171-172.

19. Pinyol M, et al. Inactivation of RB1 in mantle-cell lymphoma detected by nonsense-mediated mRNA decay pathway inhibition and microarray analysis. Blood. 2007;109(12):5422-5429.

20. Beltran E, et al. A cyclin-D1 interaction with BAX underlies its oncogenic role and potential as a therapeutic target in mantle cell lymphoma. Proc Nat Acad Sci U S A. 2011;108(30):12461-12466.

21. Hernandez L, et al. p53 gene mutations and protein overexpression are associated with aggressive variants of mantle cell lymphomas. Blood. 1996;
87(8):3351-3359

22. Hernandez L, et al. CDK4 and MDM2 gene alterations mainly occur in highly proliferative and aggressive mantle cell lymphomas with wild-type INK4a/ ARF locus. Cancer Res. 2005;65(6):2199-2206.

23 . Rosenwald A, et al. The proliferation gene expression signature is a quantitative integrator of oncogenic events that predicts survival in mantle cell lymphoma. Cancer Cell. 2003;3(2):185-197.

24. Royo C, Salaverria I, Hartmann EM, Rosenwald A, Campo E, Bea S. The complex landscape of genetic alterations in mantle cell lymphoma. Semin Cancer Biol. 2011;21(5):322-334.

25. Camacho E, et al. ATM gene inactivation in mantle cell lymphoma mainly occurs by truncating mutations and missense mutations involving the phosphatidylinositol-3 kinase domain and is associated with increasing numbers of chromosomal imbalances. Blood. 2002;99(1):238-244.

26. Hartmann EM, et al. Pathway discovery in mantle cell lymphoma by integrated analysis of high-resolution gene expression and copy number profiling. Blood. 2010;116(6):953-961.

27. Kridel R, et al. Whole transcriptome sequencing reveals recurrent NOTCH1 mutations in mantle cell lymphoma. Blood. 2012;119(9):1963-1971.

28. Puente XS, et al. Whole-genome sequencing identifies recurrent mutations in chronic lymphocytic leukaemia. Nature. 2011;475(7354):101-105.

29. Fabbri G, et al. Analysis of the chronic lymphocytic leukemia coding genome: role of NOTCH1 mutational activation. J Exp Med. 2011;208(7):1389-1401.

30. Gesk S, et al. A chromosomal translocation in cyclin D1-negative/cyclin D2-positive mantle cell lymphoma fuses the CCND2 gene to the IGK locus. Blood. 2006;108(3):1109-1110.

31. Carvajal-Cuenca A, et al. Clinicopathologic characterization of cyclin D1-negative mantle cell lymphoma. Mod Pathol. 2012;25:1370.

32. Ek S, Dictor M, Jerkeman M, Jirstrom K, Borrebaeck CA. Nuclear expression of the non B-cell lineage Sox11 transcription factor identifies mantle cell lymphoma. Blood. 2008;111(2):800-805.

33. Mozos A, et al. SOX11 expression is highly specific for mantle cell lymphoma and identifies the cyclin D1-negative subtype. Haematologica. 2009; 94(11):1555-1562.

34. Vegliante MC, et al. Epigenetic activation of SOX11 in lymphoid neoplasms by histone modifications. PLoS One. 2011;6(6):e21382.

35. Dal Col J, et al. Distinct functional significance of Akt and mTOR constitutive activation in mantle cell lymphoma. Blood. 2008;111(10):5142-5151.

36. Rizzatti EG, et al. Gene expression profiling of mantle cell lymphoma cells reveals aberrant expression of genes from the PI3K-AKT, WNT and TGFbeta signalling pathways. Br J Haematol. 2005; 130(4):516-526.

37. Rudelius $\mathrm{M}$, et al. Constitutive activation of Akt contributes to the pathogenesis and survival of mantle cell lymphoma. Blood. 2006;108(5):1668-1676.

38. Navarro A, et al. MicroRNA expression, chromosomal alterations, and immunoglobulin variable heavy chain hypermutations in Mantle cell lymphomas. Cancer Res. 2009;69(17):7071-7078.

39. Rao E, et al. The miRNA-17 approximately 92 cluster mediates chemoresistance and enhances tumor growth in mantle cell lymphoma via PI3K/AKT pathway activation. Lenkemia. 2012;26(5):1064-1072.

40. Rinaldi A, et al. Genomic and expression profiling identifies the B-cell associated tyrosine kinase Syk as a possible therapeutic target in mantle cell lymphoma. Br J Haematol. 2006;132(3):303-316.

41. Psyrri A, et al. Phosphatidylinositol 3 '-kinase catalytic subunit alpha gene amplification contributes to the pathogenesis of mantle cell lymphoma. Clin Cancer Res. 2009;15(18):5724-5732.

42. Pighi $\mathrm{C}$, et al. Phospho-proteomic analysis of man- tle cell lymphoma cells suggests a pro-survival role of B-cell receptor signaling. Cell Oncol (Dordr). 2011; 34(2):141-153.

43. Baran-Marszak F, et al. Constitutive and B-cell receptor-induced activation of STAT3 are important signaling pathways targeted by bortezomib in leukemic mantle cell lymphoma. Haematologica. 2010; 95(11):1865-1872.

44. Lai R, Rassidakis GZ, Medeiros LJ, Leventaki V, Keating M, McDonnell TJ. Expression of STAT3 and its phosphorylated forms in mantle cell lymphoma cell lines and tumours. J Pathol. 2003; 199(1):84-89.

45. Martinez N, et al. The molecular signature of mantle cell lymphoma reveals multiple signals favoring cell survival. Cancer Res. 2003;63(23):8226-8232.

46. Visser HP, Tewis M, Willemze R, Kluin-Nelemans JC. Mantle cell lymphoma proliferates upon IL-10 in the CD40 system. Leukemia. 2000;14(8):1483-1489.

47. Mottok A, et al. Inactivating SOCS1 mutations are caused by aberrant somatic hypermutation and restricted to a subset of B-cell lymphoma entities. Blood. 2009; 114(20):4503-4506.

48. Andersen NS, et al. Soluble CD40 ligand induces selective proliferation of lymphoma cells in primary mantle cell lymphoma cell cultures. Blood. 2000;96(6):2219-2225.

49. Burger JA, Ford RJ. The microenvironment in mantle cell lymphoma: cellular and molecular pathways and emerging targeted therapies. Semin Cancer Biol. 2011;21(5):308-312.

50. Kurtova AV, Tamayo AT, Ford RJ, Burger JA. Mantle cell lymphoma cells express high levels of CXCR4, CXCR5, and VLA-4 (CD49d): importance for interactions with the stromal microenvironment and specific targeting. Blood. 2009;113(19):4604-4613.

51. Gelebart P, Zak Z, en-Bard J, Anand M, Lai R. Interleukin 22 signaling promotes cell growth in mantle cell lymphoma. Transl Oncol. 2011;4(1):9-19.

52. Roue G, Perez-Galan P, Lopez-Guerra M, Villamor $\mathrm{N}$, Campo E, Colomer D. Selective inhibition of IkappaB kinase sensitizes mantle cell lymphoma $\mathrm{B}$ cells to TRAIL by decreasing cellular FLIP level. J Immunol. 2007;178(3):1923-1930.

53. Pham L V, Tamayo AT, Yoshimura LC, Lo P, Ford RJ. Inhibition of constitutive NF-kappa B activation in mantle cell lymphoma B cells leads to induction of cell cycle arrest and apoptosis. J Immunol. 2003;171(1):88-95.

54. Honma K, et al. TNFAIP3/A20 functions as a novel tumor suppressor gene in several subtypes of non-Hodgkin lymphomas. Blood. 2009; 114(12):2467-2475.

55. Gelebart P, et al. Constitutive activation of the Wnt canonical pathway in mantle cell lymphoma. Blood. 2008;112(13):5171-5179.

56. Martin P, et al. Outcome of deferred initial therapy in mantle-cell lymphoma. J Clin Oncol. 2009; 27(8):1209-1213.

57. Eve HE, Furtado MV, Hamon MD, Rule SA. Time to treatment does not influence overall survival in newly diagnosed mantle-cell lymphoma. J Clin Oncol. 2009;27(32):e189-e190.

58. Majlis A, Pugh WC, Rodriguez MA, Benedict WF, Cabanillas F. Mantle cell lymphoma: correlation of clinical outcome and biologic features with three histologic variants. J Clin Oncol. 1997;15(4):1664-1671.

59. Tiemann M, et al. Histopathology, cell proliferation indices and clinical outcome in 304 patients with mantle cell lymphoma (MCL): a clinicopathological study from the European MCL Network. $\mathrm{Br}$ J Haematol. 2005;131(1):29-38.

60. Leitch HA, Gascoyne RD, Chhanabhai M, Voss NJ, Klasa R, Connors JM. Limited-stage mantle-cell lymphoma. Ann Oncol. 2003;14(10):1555-1561.

61. Nygren L, Baumgartner Wennerholm S, Klimkowska M, Christensson B, Kimby E, Sander B. Prognostic role of SOX11 in a population-based cohort of man- 
tle cell lymphoma. Blood. 2012;119(18):4215-4223.

62 . Orchard J, et al. A subset of $\mathrm{t}(11 ; 14)$ lymphoma with mantle cell features displays mutated $\mathrm{IgVH}$ genes and includes patients with good prognosis, nonnodal disease. Blood. 2003;101(12):4975-4981.

63. Del Giudice I, et al. Behind the scenes of non-nodal MCL: downmodulation of genes involved in actin cytoskeleton organization, cell projection, cell adhesion, tumour invasion, TP53 pathway and mutated status of immunoglobulin heavy chain genes. Br J Haematol. 2012;156(5):601-611.

64. Royo C, et al. Non-nodal type of mantle cell lymphoma is a specific biological and clinical subgroup of the disease. Leukemia. 2012;26(8):1895-1898.

65. Fernandez V, et al. Genomic and gene expression profiling defines indolent forms of mantle cell lymphoma. Cancer Res. 2010;70(4):1408-1418.

66. Ondrejka SL, Lai R, Kumar N, Smith SD, Hsi ED. Indolent mantle cell leukemia: clinicopathologic variant characterized by isolated lymphocytosis, interstitial bone marrow involvement, kappa light chain restriction, and good prognosis. Haemato logica. 2011;96(8):1121-1127.

67. Rule SA, Poplar S, Evans PA, O'Connor SJ, Owen RG. Indolent mantle-cell lymphoma: immunoglobulin variable region heavy chain sequence analysis reveals evidence of disease 10 years prior to symptomatic clinical presentation. J Clin Oncol. 2011;29(15):e437-e439.

68. Navarro A, et al. Molecular subsets of mantle cell lymphoma defined by the IGHV mutational status and SOX11 expression have distinct biological and clinical features [published on line ahead of print Aug 20, 2012]. Cancer Res. doi:10.1158/0008-5472. CAN-12-1615.

69. Angelopoulou MK, et al. The splenic form of mantle cell lymphoma. Eur J Haematol. 2002;68(1):12-21.

70. Lecluse Y, Lebailly P, Roulland S, Gac AC, Nadel B, Gauduchon P. $\mathrm{t}(11 ; 14)$-positive clones can persist over a long period of time in the peripheral blood of healthy individuals. Lenkemia. 2009;23(6):1190-1193.

71. Christian B, et al. Mantle cell lymphoma 12 years after allogeneic bone marrow transplantation occurring simultaneously in recipient and donor. J Clin Oncol. 2010;28(31):e629-e632.

72. Carvajal-Cuenca A, et al. In situ mantle cell lymphoma: clinical implications of an incidental finding with indolent clinical behavior. Haematologica. 2012;97(2):270-278.

73. Racke F, Simpson S, Christian B, Blum KA, Hasserjian R, Zhao W. Evidence of long latency periods prior to development of mantle cell lymphoma. Blood. 2010;116:2834.

74. Smith MR. Should there be a standard therapy for mantle cell lymphoma? Future Oncol. 2011; $7(2): 227-237$.

75. Dreyling M. Therapy of mantle cell lymphoma: new treatment options in an old disease or vice versa? Semin Hematol. 2011;48(3):145-147.

76. Kane RC, et al. Bortezomib for the treatment of mantle cell lymphoma. Clin Cancer Res. 2007;13(18 pt 1):5291-5294

77. European Medicines Agency. Committee for medicinal products for human use post-authorisation summary of positive opinion for Torisel. Available at: http://www.emea.europa.eu/docs/ en_GB/document_library/Summary_of_opinion/ human/000799/WC500059409.pdf. Accessed on July 1, 2012.

78. Molineaux SM. Molecular pathways: targeting proteasomal protein degradation in cancer. Clin Cancer Res. 2012;18(1):15-20.

79. Perez-Galan P, Roue G, Villamor N, Montserrat E, Campo E, Colomer D. The proteasome inhibitor bortezomib induces apoptosis in mantle-cell lymphoma through generation of ROS and Noxa activation independent of p53 status. Blood. 2006; 107(1):257-264.

80. Perez-Galan P, et al. Bortezomib resistance in mantle cell lymphoma is associated with plasmacytic differentiation. Blood. 2010;117(2):542-552.

81. Mato AR, Feldman T, Goy A. Proteasome inhibition and combination therapy for non-Hodgkin's lymphoma: from bench to bedside. Oncologist. 2012; 17(5):694-707.

82. Parekh S, Weniger MA, Wiestner A. New molecular targets in mantle cell lymphoma. Semin Cancer Biol. 2011;21(5):335-346.

83. Georgakis GV, Li Y, Younes A. The heat shock protein 90 inhibitor 17-AAG induces cell cycle arrest and apoptosis in mantle cell lymphoma cell lines by depleting cyclin D1, Akt, Bid and activating caspase 9. Br J Haematol. 2006;135(1):68-71.

84. Kirschbaum M, et al. Phase II study of vorinostat for treatment of relapsed or refractory indolent non-Hodgkin's lymphoma and mantle cell lymphoma. J Clin Oncol. 2011;29(9):1198-1203.

85. Dancey J. mTOR signaling and drug development in cancer. Nat Rev Clin Oncol. 2010;7(4):209-219.

86 . Hess J, et al. Phase III study to evaluate temsirolimus compared with investigator's choice therapy for the treatment of relapsed or refractory mantle cell lymphoma. J Clin Oncol. 2009;27(23):3822-3829.

87. Ansell SM, et al. Low-dose, single-agent temsirolimus for relapsed mantle cell lymphoma: a phase 2 trial in the North Central Cancer Treatment Group. Cancer. 2008;113(3):508-514.

88. Ansell SM, et al. Temsirolimus and rituximab in patients with relapsed or refractory mantle cell lymphoma: a phase 2 study. Lancet Oncol. 2011; 12(4):361-368.

89. Hess G, et al. Phase III study to evaluate temsirolimus compared with investigator's choice therapy for the treatment of relapsed or refractory mantle cell lymphoma. J Clin Oncol. 2009;27(23):3822-3829.

90. Hess $G$. Temsirolimus for the treatment of mantle cell lymphoma. Expert Rev Hematol. 2009;2(6):631-640.

91. Witzig TE, et al. Phase II trial of single-agent temsirolimus (CCI-779) for relapsed mantle cell lymphoma. J Clin Oncol. 2005;23(23):5347-5356.

92. Wang L, et al. Cytostatic and anti-angiogenic effects of temsirolimus in refractory mantle cell lymphoma. J Hematol Oncol. 2010;3:30.

93. Renner C, et al. A multicenter phase II trial (SAKK 36/06) of single-agent Everolimus(RAD001) in patients with relapsed or refractory mantle cell lymphoma. Haematologica. 2012;97(7):1085-1091.

94. Rizzieri DA, et al. A phase 2 clinical trial of deforolimus (AP23573, MK-8669), a novel mammalian target of rapamycin inhibitor, in patients with relapsed or refractory hematologic malignancies. Clin Cancer Res. 2008;14(9):2756-2762.

95. Kahl B, et al. Clinical safety and activity in a phase 1 study of CAL-101, an isoform-selective inhibitor of phosphatidylinositol 3-kinase P110\{delta\}, in patients with relapsed or refractory non-Hodgkin lymphoma. Blood. 2010;116:1777.

96. Gupta M, et al. Dual mTORC1/mTORC2 inhibition diminishes Akt activation and induces Pumadependent apoptosis in lymphoid malignancies. Blood. 2012;119(2):476-487.

97. Bea $S$, et al. Increased number of chromosomal imbalances and high-level DNA amplifications in mantle cell lymphoma are associated with blastoid variants. Blood. 1999;93(12):4365-4374.

98. Pinyol M, et al. Deletions and loss of expression of p16INK4a and p21Waf1 genes are associated with aggressive variants of mantle cell lymphomas. Blood. 1997;89(1):272-280.
99. Kouroukis CT, et al. Flavopiridol in untreated or relapsed mantle-cell lymphoma: Results of a phase II study of the National Cancer Institute of Canada Clinical Trials Group. J Clin Oncol. 2003; 21(9):1740-1745

100. Leonard JP, et al. Selective CDK4/6 inhibition with tumor responses by PD0332991 in patients with mantle cell lymphoma. Blood. 2012;119(20):4597-4607.

101. Greiner TC, et al. Mutation and genomic deletion status of ataxia telangiectasia mutated (ATM) and p53 confer specific gene expression profiles in mantle cell lymphoma. Proc Natl Acad Sci U S A. 2006; 103(7):2352-2357.

102. Golla RM, et al. Inhibition of poly(ADP-ribose) polymerase (PARP) and ataxia telangiectasia mutated (ATM) on the chemosensitivity of mantle cell lymphoma to agents that induce DNA strand breaks [published online ahead of print December 14, 2011]. Hematol Oncol. doi:10.1002/hon.1020.

103. Weston VJ, et al. The PARP inhibitor olaparib induces significant killing of ATM-deficient lymphoid tumor cells in vitro and in vivo. Blood. 2010; 116(22):4578-4587.

104. Williamson CT, et al. ATM deficiency sensitizes mantle cell lymphoma cells to poly(ADP-ribose) polymerase-1 inhibitors. Mol Cancer Ther. 2010; 9(2):347-357.

105. Vogler M, Dinsdale D, Dyer MJ, Cohen GM. Bcl-2 inhibitors: small molecules with a big impact on cancer therapy. Cell Death Differ. 2009;16(3):360-367.

106.Paoluzzi L, et al. The BH3-only mimetic ABT-737 synergizes the antineoplastic activity of proteasome inhibitors in lymphoid malignancies. Blood. 2008;112(7):2906-2916.

107. Perez-Galan P, Roue G, Villamor N, Campo E, Colomer D. The BH3-mimetic GX15-070 synergizes with bortezomib in mantle cell lymphoma by enhancing Noxa-mediated activation of Bak. Blood. 2007;109(10):4441-4449.

108. Vogler M, et al. A novel paradigm for rapid ABT737-induced apoptosis involving outer mitochondrial membrane rupture in primary leukemia and lymphoma cells. Cell Death Differ. 2008;15(5):820-830.

109. Wilson WH, et al. Navitoclax, a targeted high-affinity inhibitor of BCL-2, in lymphoid malignancies: a phase 1 dose-escalation study of safety, pharmacokinetics, pharmacodynamics, and antitumour activity. Lancet Oncol. 2010;11(12):1149-1159.

110. Ysebaert L, Morschhauser F. Enzastaurin hydrochloride for lymphoma: reassessing the results of clinical trials in light of recent advances in the biology of B-cell malignancies. Expert Opin Investig Drugs. 2011;20(8):1167-1174.

111. Friedberg JW, et al. Inhibition of Syk with fostamatinib disodium has significant clinical activity in non-Hodgkin lymphoma and chronic lymphocytic leukemia. Blood. 2010;115(13):2578-2585.

112. Wang L, et al. The Bruton's tyrosine kinase inhibitor PCI-32765 is highly active as single-agent therapy in previously-treated Mantle Cell Lymphoma (MCL): preliminary results of a phase II trial. Blood. 2011;118:442.

113. Younes A, Fanale MA, McLaughlin P, Copeland A, Zhu J, de Castro Faria S. Phase I study of a novel oral JAK-2 inhibitor SB1518 in patients with relapsed lymphoma: evidence of clinical and biologic activity in multiple lymphoma subtypes. Blood. 2010;116:2830.

114. Wang $Z$, et al. Targeting Notch signaling pathway to overcome drug resistance for cancer therapy. Biochim Biophys Acta. 2010;1806(2):258-267.

115.Palomero T, Ferrando A. Therapeutic targeting of NOTCH1 signaling in T-cell acute lymphoblastic leukemia. Clin Lymphoma Myeloma. 2009; 9(suppl 3):S205-S210. 